\title{
Intracellular Flow in Optic Nerve Axons: A Mechanism for Cell Death in Glaucoma
}

\author{
Leab R. Band, ${ }^{* 1,2}$ Cameron L. Hall, ${ }^{3,4}$ Giles Richardson, ${ }^{5}$ Oliver E. Jensen, ${ }^{1}$ \\ Jennifer H. Siggers, ${ }^{6}$ and Alexander J. E. Foss ${ }^{*, 7}$
}

Purpose. In glaucoma, elevated intraocular pressure causes a progressive loss of retinal ganglion cells and results in optic neuropathy. The authors propose a potential mechanism for cell death, whereby elevated intraocular pressure causes fluid to permeate axonal membranes, creating a passive intracellular fluid flow within the axons. It is hypothesized that this intracellular flow locally depletes the adenosine triphosphate (ATP) concentration, disrupting axonal transport and leading to cell death.

Methods. A mathematical model was developed that takes into account the biomechanical principles underpinning the proposed hypothesis, and was solved to determine the implications of the mechanism.

Results. The model suggests that the raised intraocular pressures present in glaucoma are adequate to produce significant intracellular fluid flow. In the periphery of the optic nerve head, this flow may be sufficient to disrupt the diffusion of ATP and hence interrupt active axonal transport.

Conclusions. The mathematical model demonstrates that it is physically plausible that a passive intracellular fluid flow could significantly contribute to the pathophysiology of the retinal ganglion cell axon in glaucoma. (Invest Ophthalmol Vis Sci. 2009;50:3750 -3758) DOI:10.1167/iovs.08-2396

$\mathrm{T}$ he glaucomas are the second commonest cause of blindness in Western countries and are characterized by raised intraocular pressure and progressive loss of retinal ganglion cells, giving rise to an optic neuropathy. ${ }^{1}$ The mechanism whereby a high intraocular pressure leads to the loss of retinal ganglion cells has proven enigmatic. ${ }^{1-3}$ Proposed theories have concentrated on whether cell loss is due to mechanical defor-

From the ${ }^{1}$ School of Mathematical Sciences, University of Nottingham, Nottingham, United Kingdom; the ${ }^{2}$ Centre for Plant Integrative Biology, University of Nottingham, Sutton Bonington, United Kingdom; the ${ }^{3}$ School of Mathematical Sciences and the ${ }^{4}$ Institute of Health and Biomedical Innovation, Queensland University of Technology, Brisbane, Queensland, Australia; the ${ }^{5}$ School of Mathematical Sciences, University of Southampton, Southampton, United Kingdom; the ${ }^{6}$ Department of Bioengineering, Imperial College London, London, United Kingdom; and the ${ }^{7}$ Department of Ophthalmology, Queen's Medical Centre, Nottingham, United Kingdom.

This article arose from research performed during the EPSRCfunded Mathematics in Medicine Study group at the University of Nottingham in September 2006.

Supported by the U.K. Research Council, EPSRC Grant EP/ D502365/1. For further details please see http//gow.epsrc.ac.uk/ ViewGrant.aspx?Grant Ref=EP/D5023/1.

Submitted for publication June 6, 2008; revised September 22 and December 22, 2008; accepted May 19, 2009.

Disclosure: L.R. Band, None; C.L. Hall, None; G. Richardson, None; O.E. Jensen, None; J.H. Siggers, None; A.J.E. Foss, None

The publication costs of this article were defrayed in part by page charge payment. This article must therefore be marked "advertisement" in accordance with 18 U.S.C. $\$ 1734$ solely to indicate this fact.

"Each of the following is a corresponding author: (Theoretical) Leah R. Band, School of Mathematical Sciences, University of Nottingham, Nottingham, NG7 2RD, UK; leah.band@nottingham.ac.uk.

(Clinical) Alexander Foss, Department of Ophthalmology, Queen's Medical Centre, Nottingham, NG7 2UH, UK; alexander.foss@nottingham.ac.uk. mation $^{4,5}$ or to an adverse effect on vascular perfusion ${ }^{6}$; however, both these theories are problematic.

The deformation theory suggests that an increase in intraocular pressure induces mechanical stress in the load-bearing tissues of the optic nerve head (the lamina cribrosa, peripapillary sclera, and scleral canal), which causes tissue deformation. As the tissue deforms, it pinches the retinal ganglion cells inducing physiological stress that could lead to cell death. ${ }^{5}$ Experimental investigations have characterized the tissue architecture,$^{7-9}$ and finite-element methods have been used to understand the impact of an increase in intraocular pressure on the biomechanics of the optic nerve head ${ }^{5,10,11}$; however, it is not well understood how tissue deformation and remodeling affect the function of the retinal ganglion cells and whether these processes can lead to cell death.

The second postulated mechanism is vascular, rather than mechanical. The intraocular circulation, in common with most tissues, shows autoregulation (tissue perfusion being independent of pressure), ${ }^{12,13}$ and it is often stated that glaucoma is characterized by faulty autoregulation of blood flow in the optic nerve head, ${ }^{14-16}$ despite very little supporting evidence. Modest elevations of intraocular pressure in the region of 10 $\mathrm{mm} \mathrm{Hg}$ are sufficient to lead to optic neuropathy, even though this elevation is well within the range in which autoregulation will operate. ${ }^{17,18}$ It is very hard to convincingly demonstrate altered autoregulation in patients with glaucoma. ${ }^{19}$ Although optic nerve head perfusion is reduced in patients with glaucoma, this effect may not be caused by faulty autoregulation and may simply reflect a lower oxygen demand due to axonal death. Patients with intraocular hypertension (but with no glaucomatous loss of ganglion cells) have no such reduction in perfusion. ${ }^{20}$

The mechanical and vascular mechanisms of glaucoma are not mutually exclusive, nor are they the only possible causes of glaucoma. For example, it has been proposed that glaucomatous neuropathy may be initiated by mechanical or ischemic damage to the astrocytes of the optic nerve head. ${ }^{21}$ This injury is thought to lead to a weakening of the cribrosal beams as well as interfering with axon metabolism. ${ }^{21}$ Although recent evidence indicates that glaucomatous elevated intraocular pressure can lead to changes in astrocyte gene expression, ${ }^{22,23}$ further experimental work is needed to clarify the details of this promising theory. A further hypothesis concerning the etiology of glaucoma is the biomechanical paradigm, whereby the dynamic effects of the intraocular pressure and blood flow are related and the physiological susceptibility of the tissue determines its response to the given stress. ${ }^{5}$ This paradigm also allows for the possibility that part or all of the axonal injury is mediated by astrocyte damage. ${ }^{5}$

Any convincing mechanism must be able to explain the interruption in active axonal transport (AAT) that occurs. ${ }^{24-29}$ Here, AAT refers to the process whereby vesicles are transported along microtubules by the dynein and kinesin motor molecules (for review, see Vallee et al. ${ }^{30}$ ) with kinesins driving orthograde transport (cell body to synapse) and dyneins retrograde transport (synapse to cell body; Fig. 1). The dynein and kinesin motor molecules gain energy by hydrolyzing ATP (adenosine triphosphate), which is produced by the mitochon- 


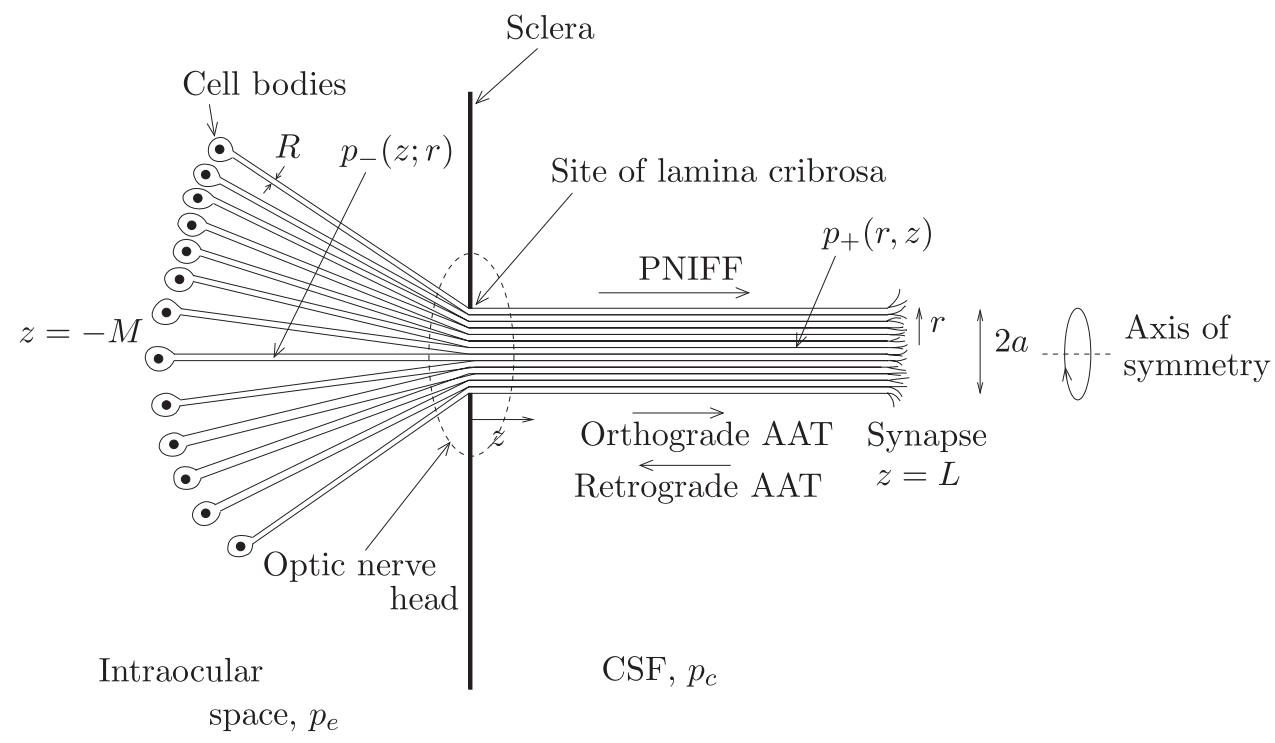

Figure 1. The axons pass from the eye into the optic nerve. In the eye, the axons are well spaced, whereas in the optic nerve the axons form a tightly packed bundle.
The axons will then not be able to maintain communication between the cell body and the synapse, which could lead to cell death.

In this theoretical study, we developed a mathematical model to show that the PNIFF mechanism is physically plausible, and used our model to investigate the pattern and likely severity of cell damage that is predicted by our hypothesis.

\section{Methods}

We consider the geometry shown in Figure 1. In the optic nerve, we use a cylindrical coordinate system $(r, z)$, where $z$ measures the distance along the axis of the optic nerve and $r$ represents the radial position of an axon within the optic nerve, where $0 \leq r<a$ and $a$ is the radius of the optic nerve. In the eye, however, the axons are dispersed throughout the retina, and we let $z$ measure the distance along the individual axons. We suppose that the lamina cribrosa has negligible thickness and define its position to be $z=0$, such that, for each axon, the region $z<0$ is in the eye while $z>0$ lies in the optic nerve. (For completeness, Appendix $\mathrm{C}$ shows that considering a finitethickness lamina cribrosa does not significantly affect the results presented.) In the eye, each axon ends at a cell body at $z=-M<0$, and in the brain the axons end at the synaptic terminal at $z=L>0$; thus, all the axons are assumed to have the same length. We suppose that the axons are sufficiently rigid that, despite changes in external pressure, they have a constant, uniform radius, $R$ (where $R \ll a$ ). There are approximately $10^{6}$ axons, ${ }^{31,32}$ and so the individual axons are sufficiently small that we can consider them as a continuum within the optic nerve. This enables us to make the assumption that the system is axisymmetric around the optic nerve's central axis $(r=0)$.

Each axon is treated as a hollow, porous tube that carries a passive axial fluid flux, $F(r, z)$ (the PNIFF), with a corresponding maximum axial velocity, $U(r, z)$. We denote the dynamic viscosity of the axoplasm as $\mu$ and the intracellular pressure in the eye region and optic nerve region as $p_{-}(z ; r)$ and $p_{+}(r, z)$, respectively, where the intracellular pressure in the eye region is parameterized by the radial position at which the axon enters the optic nerve, $r$ (i.e., $r$ labels the axons). Although in the optic nerve the intracellular pressure is strictly defined only within each axon, we represent it by a smoothly varying continuous function of the radial coordinate, $p_{+}(r, z)$, using a homogenization approximation. ${ }^{33}$ In the eye, $z \leq 0$, the axons are widely spaced so that the pressure exterior to each can be assumed to be the constant intraocular pressure, $p_{\mathrm{e}}$. In contrast, the axons in the optic nerve are tightly bundled together and so the external pressure depends on that of the surrounding axons and may be nonuniform (Fig. 1). The optic nerve is surrounded by cerebrospinal fluid (CSF) at constant pressure 
$p_{\mathrm{c}}$. The hydraulic conductivities of the axons' membranes inside the optic nerve and inside the eye are denoted $\kappa_{+}$and $\kappa_{-}$, respectively, each of which is taken to be constant.

\section{Parameter Values}

We now estimate the values of the parameters in the model. We assume that each axon's total length is $L+M=0.11 \mathrm{~m}$, of which $L=$ $0.1 \mathrm{~m}$ is within the optic nerve (extraocular) and the remaining $M=$ $0.01 \mathrm{~m}$ is in the eye (intraocular). The lamina cribrosa is typically 100 to $300 \mu \mathrm{m}$ thick. ${ }^{8,11}$ As this length scale is much less than the axon's length, we can consider the thickness of the lamina cribrosa to be negligible. In the optic nerve, the axons are bundled together resulting in an optic nerve radius $a=1.6 \mathrm{~mm} .{ }^{31}$ Physiologically, axons have different radii; estimated radii are in the range 0.05 to $4.15 \mu \mathrm{m} .{ }^{31}$ It has been suggested that thicker axons are more susceptible to injury in glaucoma, ${ }^{34-36}$ and so we consider a radius at the upper end of the quoted range and let $R=4.15 \mu \mathrm{m}$. A key parameter in the model is the axoplasm viscosity; in the mammalian nerve, measurements suggest that this viscosity is $5 \mathrm{cP}$ (or $0.005 \mathrm{~Pa} \mathrm{~s}$ ), ${ }^{37}$ which is about five times greater than that of water.

A non-zero pressure drop across the axon's membrane induces a transmural fluid flux. We characterize the membrane by its hydraulic conductivity, which is the flux induced per unit area per unit pressure drop across the membrane. As the axons are myelinated in the optic nerve but not in the eye, we use two distinct conductivities, $\kappa_{-}$in the eye and $\kappa_{+}$in the optic nerve, where $\kappa_{-}>\kappa_{+}$. Several studies have estimated $\kappa_{-}$: Spyropoulos $^{38}$ gives 8-14 $\times 10^{-8} \mathrm{~cm} \mathrm{~s}^{-1} \mathrm{~cm} \mathrm{H}_{2} \mathrm{O}^{-1}$, Vargas $^{39}$ suggests $10.6 \times 10^{-8} \mathrm{~cm} \mathrm{~s}^{-1} \mathrm{~cm} \mathrm{H}_{2} \mathrm{O}^{-1}$, and Wallin ${ }^{40}$ estimates $0.236 \times 10^{-8} \mathrm{~cm} \mathrm{~s}^{-1} \mathrm{~cm} \mathrm{H}_{2} \mathrm{O}^{-1}$. We therefore chose a representative value, $\kappa_{-}=10 \times 10^{-8} \mathrm{~cm} \mathrm{~s}^{-1} \mathrm{~cm} \mathrm{H}_{2} \mathrm{O}^{-1}=10^{-11} \mathrm{~m} \mathrm{~s}^{-1}$ $\mathrm{Pa}^{-1}$. As far as we are aware, experimental estimates for $\kappa_{+}$are not available. We assume that the myelinated sheath reduces the membrane conductivity to a tenth of its value in the nonmyelinated intraocular region.

In health, ATP is distributed throughout the axon primarily by diffusion, with the diffusion coefficient, $D=3 \times 10^{-10} \mathrm{~m}^{2} \mathrm{~s}^{-1} 41$; however, the PNIFF will advect ATP toward the synapse $(z=L)$, which could result in localized ATP depletion. To assess whether the PNIFF affects the ATP distribution, we analyze the relative strengths of ATP diffusion and advection, and so introduce a typical length scale, $l$, over which ATP diffuses. As ATP is produced by roughly evenly spaced mitochondria, it must diffuse between them to supply energy throughout the axon; therefore it is appropriate to take $l$ to be the distance between neighboring mitochondria. Published values for this length scale do not seem to be currently available, and we therefore estimate an approximate value of $l=10 \mu \mathrm{m}$.

Based on these considerations, our estimates of typical parameter values are summarized in Table 1. Later, we shall discuss how sensitive our results are to variations in the axoplasm viscosity, $\mu$, intraocular pressure, $p_{\mathrm{e}}$, and axons' radii, $R$.

\section{Governing Equations}

In each axon, Poiseuille's Law relates the PNIFF, $F(r, z)$, to the axial pressure gradient, giving

$$
F(r, z)=-\frac{\pi R^{4}}{8 \mu} \frac{\partial p}{\partial z}
$$

which corresponds to a maximum axial fluid velocity

$$
U(r, z)=\frac{2 F}{\pi R^{2}}=\frac{R^{2}}{4 \mu} \frac{\partial p}{\partial z}
$$

where the pressure $p=p_{+}(r, z)$ or $p=p_{-}(z ; r)$. Equating the rate of change of flux through each axon in the eye $(z<0)$ to the transmural flux that leaks through its walls gives

$$
-\frac{d}{d z}\left(\frac{R^{4} \pi}{8 \mu} \frac{d p_{-}}{d z}\right)+2 \pi R \kappa_{-}\left(p_{-}-p_{\mathrm{e}}\right)=0 \quad \text { for } \quad z<0
$$

The leakage is driven by the transmural pressure difference $p_{-}(z ; r)-$ $p_{\mathrm{e}}$. Osmotic pressure gradients between the axoplasm and the intraocular space are neglected. We note that the dependence of $p_{-}$on $r$ in $z<0$ is only parametric, allowing us to use ordinary $z-$ derivatives in equation 3 .

The situation is somewhat more complicated in the optic nerve $(z>0)$. We assume that the axons are tightly packed so that fluid leaks directly from one axon into its neighbors rather than into the interstitial space (which we take to be of negligible volume). In this instance, the pressure in each axon is governed by

$$
\begin{aligned}
&-\frac{\partial}{\partial z}\left(\frac{\sqrt{3} R^{4}}{4 \mu} \frac{\partial p_{+}}{\partial z}\right)-\frac{2 \sqrt{3} R^{3} \kappa_{+}}{r} \frac{\partial}{\partial r}\left(r \frac{\partial p_{+}}{\partial r}\right)=0 \\
& \text { for } \quad 0<r<a, \quad z>0
\end{aligned}
$$

(see Appendix A for further explanation). In this case, the pressure difference between neighboring axons (represented by the radial pressure gradient $\partial p_{+} / \partial r$ ) drives transmural fluxes from axon to axon.

We assume that the optic nerve is axisymmetric and that the pressure is continuous at the edge of the optic nerve; therefore

$$
\frac{\partial p_{+}}{\partial r}=0 \quad \text { on } \quad r=0, \quad p_{+}=p_{c} \quad \text { on } \quad r=a
$$

(for $z>0$ ). Although we expect a small pressure drop across the membranes enclosing the optic nerve's peripheral axons, preliminary investigations suggested that continuity of pressure at the edge of the optic nerve, equation $5 \mathrm{~b}$, is an appropriate simplification. We assume that no axoplasm enters or leaves through the ends of the axons, so that

$$
F=0 \quad \text { at } \quad z=L,-M \text {. }
$$

Finally, continuity of pressure and flux across the lamina cribrosa provide conditions on $z=0$ (for all $0 \leq r<a$ )

TABLE 1. Physiological Estimates of the Dimensional Parameters Required by the Model

\begin{tabular}{llcc}
\hline Parameter & \multicolumn{1}{c}{ Descriptions } & Value & Ref. \\
\hline$R$ & Axon radius & $4.15 \times 10^{-6} \mathrm{~m}$ & 31 \\
$a$ & Optic-nerve radius & $1.6 \times 10^{-3} \mathrm{~m}$ & 31 \\
$\mu$ & Axoplasm viscosity & $0.005 \mathrm{~Pa} \mathrm{~s}$ & 37 \\
$\kappa_{-}$ & Axon membrane conductivity in the eye & $10^{-11} \mathrm{~ms}^{-1} \mathrm{~Pa}^{-1}$ & 38,39 \\
$\kappa_{+}$ & Axon membrane conductivity in the optic nerve & $10^{-12} \mathrm{~ms}^{-1} \mathrm{~Pa}^{-1}$ & \\
$p_{\mathrm{e}}$ & Intraocular pressure (30 mm Hg) & $4.0 \times 10^{3} \mathrm{~Pa}$ & \\
$p_{\mathrm{c}}$ & CSF pressure (10 mm Hg) & $1.3 \times 10^{3} \mathrm{~Pa}$ \\
$M$ & Length of axon in the eye & $0.01 \mathrm{~m}$ & \\
$L$ & Length of axon in the optic nerve & $0.10 \mathrm{~m}$ & \\
$l$ & Diffusive length scale for ATP & $3 \times 10^{-5} \mathrm{~m} \mathrm{~m}^{2} \mathrm{~s}^{-1}$ & 41 \\
$D$ & Diffusion coefficient for ATP & &
\end{tabular}




$$
p_{-}=p_{+}, \quad \frac{d p_{-}}{d z}=\frac{\partial p_{+}}{\partial z} \quad \text { at } \quad z=0
$$

In Appendix B, we nondimensionalize the governing equations 3 and 4 and boundary conditions (equations 5-7), and solve for the axoplasmic pressures, $p_{-}(z ; r)$ and $p_{+}(r, z)$, using a series of Bessel functions.

\section{Results}

Figure 2 shows the intracellular pressure and flux in the axons within the eye with an intraocular pressure of $30 \mathrm{~mm} \mathrm{Hg}$ (calculated from our Bessel-function solution). In the axons that enter the optic nerve near its center, the intracellular pressure is approximately constant and equal to the intraocular pressure, resulting in no intracellular flux. However, in those that enter near the edge of the optic nerve, the intracellular
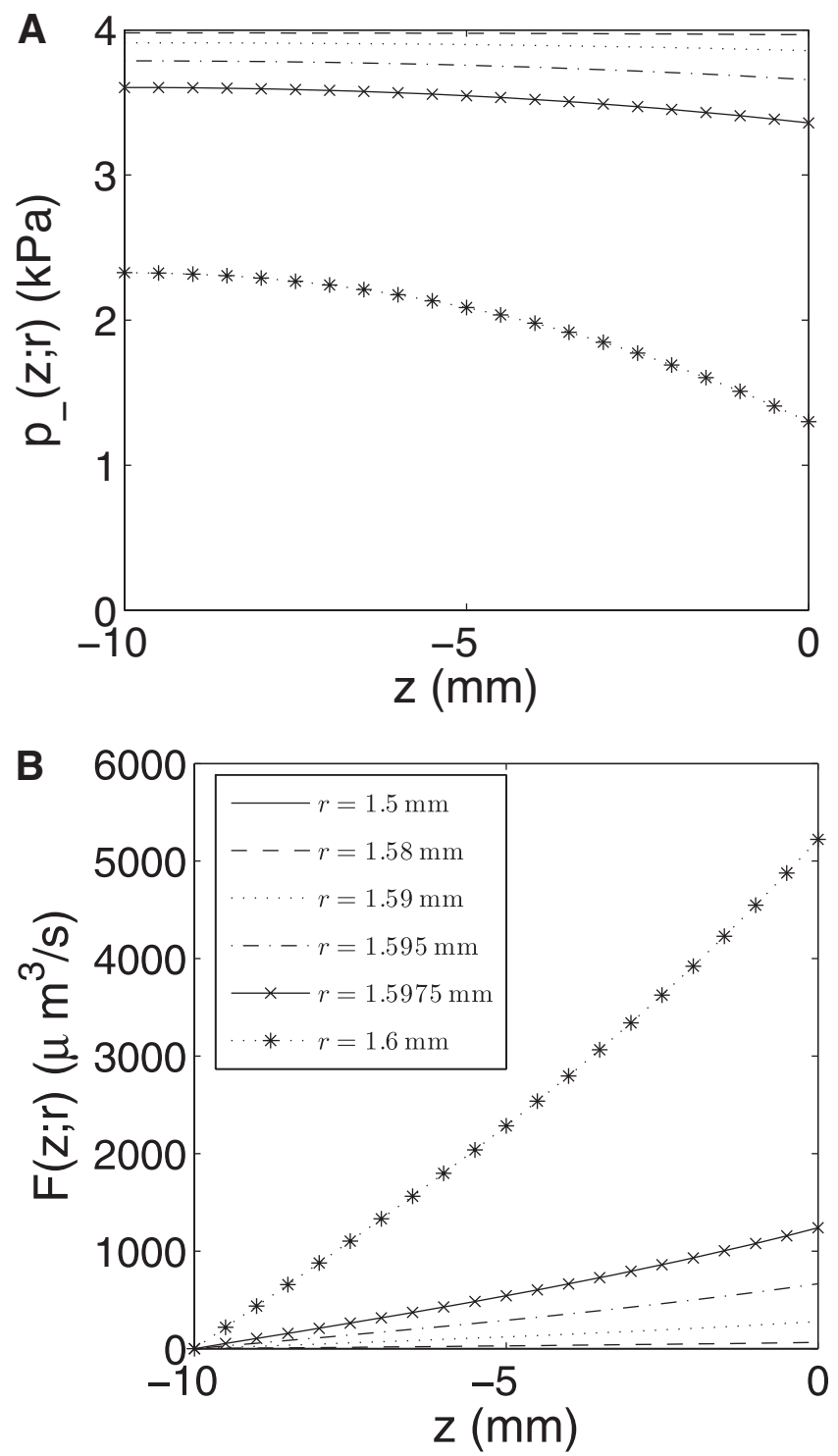

Figure 2. The flow dynamics in the axons within the eye, with an intraocular pressure of $p_{\mathrm{e}}=30 \mathrm{~mm} \mathrm{Hg} \approx 4.0 \mathrm{kPa}$ and the parameter estimates given in Table 1. The edge of the optic nerve is at $r=1.6$ $\mathrm{mm}$; only the dynamics close to the edge of the optic nerve are shown, because the pressure and flux are approximately uniform in the central bulk of the optic nerve. (A) Intracellular pressure, $p_{-}(z ; r)$ (in kilopascals); (B) PNIFF, $F(z ; r)$ (in cubic micrometers/second).
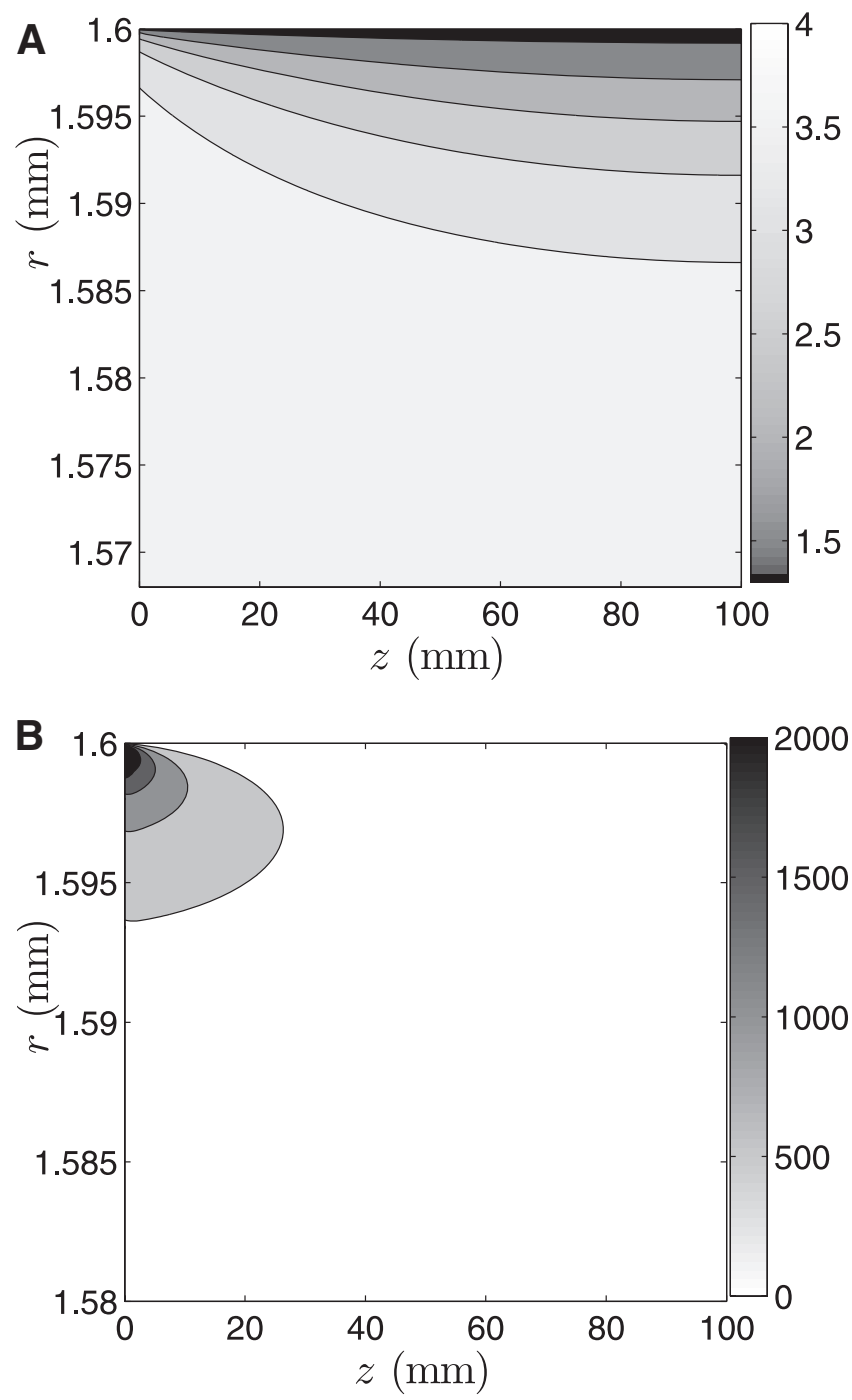

FIGURE 3. Pressure and flux in the optic nerve axons, with an intraocular pressure of $p_{\mathrm{e}}=30 \mathrm{~mm} \mathrm{Hg} \approx 4.0 \mathrm{kPa}$ and the parameter estimates given in Table 1. The edge of the optic nerve is at $r=1.6 \mathrm{~mm}$, and only the region close to the edge of the optic nerve is shown, because the pressure and flux are approximately uniform in the central bulk of the optic nerve. Contour plots show (A) the axoplasmic pressure, $p_{+}(r, z)$ (in kilopascals) and (B) the PNIFF, $F(r, z)$ (in cubic micrometers/second).

pressure is lower and reduces toward the CSF pressure close to the optic nerve $(z=0)$, which results in an intracellular flux through the axons. The corresponding pressure and flux within the region at the edge of the optic nerve are shown in Figure 3. In the central bulk of the optic nerve, the axoplasmic pressure equals the elevated intraocular pressure, $p_{\mathrm{e}}$, whereas at its periphery, the axoplasmic pressure rapidly reduces to the CSF pressure, $p_{\mathrm{c}}$. This gives rise to large radial pressure gradients at the periphery of the optic nerve, that in turn lead to a significant axial flux (the PNIFF) in the peripheral axons. The PNIFF depends on the difference between the intraocular and CSF pressures and not on the absolute values of these pressures. The model predicts that a larger pressure difference creates a larger pressure gradient and therefore a larger PNIFF.

In turn, we expect the PNIFF to influence the axoplasmic ATP distribution. In health, ATP is distributed throughout the axon primarily by diffusion; however, the PNIFF will advect ATP, which will result in some localized ATP depletion. The relative importance of advective to diffusive motion is characterized by the Péclet number, which has the formula $U l / D$, 
where $U(r, z)$ is the maximum axial velocity of the PNIFF suggested by the model (see Leal ${ }^{42}$ for further details). The Péclet number depends on the parameters $l$ and $D$; we note that the calculated Péclet number is smaller if we use a smaller estimate of $l$ or a larger estimate of $D$. A large Péclet number indicates that advection dominates over diffusion, meaning that there will be significant ATP depletion. The transition from diffusion-dominated to advection-dominated is not strict as the Péclet number increases, but it occurs when the Péclet number is around 1. In Figure 4, we consider several physiological pressure differences (intraocular - CSF), and identify the regions of the optic nerve in which we expect ATP depletion to be most severe, leading to interruption of AAT and cell death. Thus, we predict that AAT is interrupted in a single layer of axons at the periphery of the optic nerve (although the continuum approximation may be inappropriate on such length scales, a discrete model would predict the same behavior and would be computationally intensive). Once the peripheral axons die, the PNIFF will affect the adjacent layer of axons, resulting in a slow progression of optic pathophysiology. With a larger pressure difference between the intraocular fluid and the CSF, we predict a larger region in which advection is likely to cause an ATP deficiency, and this in turn increases the number of cells affected.

As discussed earlier, parameter estimates vary between different sources. With a higher viscosity estimate, the model predicts a smaller pressure gradient and PNIFF; however, although the model is sensitive to the value of the viscosity, all available estimates ${ }^{43-49}$ still result in significant PNIFF close to the edge of the optic nerve. The PNIFF also depends on the axons' radii, $R$; considering a larger value of the radii, $R$, results in a larger PNIFF, which suggests that glaucoma is more prevalent in thicker axons.

\section{Discussion}

There are two groups of existing theories for the mechanism of optic neuropathy in patients with glaucoma: the mechanical and the vascular. We propose a third theory based on a passive neuronal intracellular fluid flux (PNIFF) in the optic nerve axons. The mathematical model presented herein demonstrates the plausibility of the PNIFF mechanism and suggests that typical glaucomatous pressure differences between the intraocular fluid and CSF are sufficient to create significant PNIFF in the axons at the edge of the optic nerve close to the lamina cribrosa. The PNIFF advects ATP molecules, creating a region of ATP depletion, which we propose leads to a breakdown of AAT. Although the PNIFF may transport material that could compensate for the breakdown of orthograde AAT, retrograde transport would still be impaired. The locations of reduced AAT in the experiments reported in

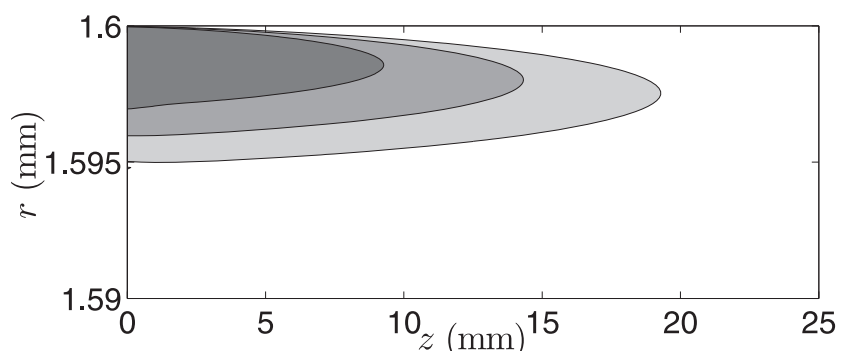

FIGURE 4. Shaded areas indicate the region of the optic nerve in which the Péclet number is greater than one, indicating that advection is likely to dominate over diffusion. Three values of the pressure difference (intraocular $-\mathrm{CSF}$ ) are shown: $p_{\mathrm{e}}-p_{\mathrm{c}}=25 \mathrm{~mm} \mathrm{Hg} \approx 3.33 \mathrm{kPa}$ (light gray), $p_{\mathrm{e}}-p_{\mathrm{c}}=20 \mathrm{~mm} \mathrm{Hg} \approx 2.66 \mathrm{kPa}$ (medium gray) and $p_{\mathrm{e}}-p_{\mathrm{c}}=$ $15 \mathrm{~mm} \mathrm{Hg} \approx 2 \mathrm{kPa}$ (dark gray). The theory is that ATP depletion is most severe in the shaded regions, leading to potential cell death. references $^{24-29}$ (described in the introduction) are consistent with our findings.

As discussed in the introduction, there is excellent experimental evidence to support failure of AAT in the retinal ganglion cells in glaucoma. Failure of AAT has been implicated in a number of neurodegenerative conditions including Alzheimer's and Huntingdon's diseases (see Ref. 50 for review), and mutations in kinesin family members have been found in neuropathies such as Charcot-Marie-Tooth ${ }^{51}$ and hereditary spastic paraplegia. ${ }^{52}$ This evidence supports the hypothesis that interruption of AAT can lead to neuronal death. Orthograde axonal transport is essential because the mRNA required for protein synthesis occurs in the cell body; therefore either it or the translated proteins have to travel the length of the axon to maintain the integrity of the cell synapse. ${ }^{53}$ Retrograde transport is important for cell maintenance, for example, by transporting brain-derived neurotrophic factor. ${ }^{54,55}$ Thus, blocking either orthograde or retrograde transport is potentially fatal to retinal ganglion cells, although to date no such mechanism has been linked to optic neuropathies. ${ }^{56}$

The model predicts that it is the location of the axons within the optic nerve at the level of the lamina cribrosa that determines their sensitivity to damage. In agreement with some experimental findings, ${ }^{24-29}$ the model suggests that AAT will be interrupted in peripheral axons, which may explain why increased intraocular pressure appears to particularly affect the peripheral visual field and the Bjerrum area. Although the axons in the Bjerrum area run in the center of the optic nerve, they pass the lamina cribrosa in the optic nerve's periphery, and in agreement with the model's predictions, are thought to be particularly vulnerable to glaucomatous damage. ${ }^{57}$ The PNIFF mechanism also suggests that thicker axons are more susceptible to glaucomatous damage, which is also in agreement with some experimental findings. ${ }^{34-36}$ Spatial variations in the axons' radii within the optic nerve ${ }^{31,32}$ would influence the pattern of optic pathophysiology (we could consider these spatial variations in an extended model by letting the axons' radii depend on $\theta$ and $r$ ).

An important feature of the model is that the key parameter is not the absolute value of the intraocular pressure, but rather the difference between the intraocular and CSF pressures. There are many similarities in the way intraocular fluid and CSF are produced, and in health, the two pressures are strongly correlated $(r=0.96) .{ }^{58}$ Clinically, there is no noninvasive way to measure CSF pressure, and so glaucoma is normally assessed on intraocular pressure measurements alone; however, there is a poor correlation between glaucomatous optic neuropathy progression and intraocular pressure, ${ }^{59-62}$ and a recent clinical study $^{63}$ observed low CSF pressures in patients with glaucoma. This finding supports the model's hypothesis that it is the pressure difference between the intraocular fluid and CSF (rather than the absolute value of the intraocular pressure) that leads to glaucomatous damage of retinal ganglion cell axons. Thus, although it has been suggested that activities that raise intraocular pressure, such as exercise and wearing a tight neck tie, may increase the likelihood of glaucoma ${ }^{64}$ these activities raise the CSF pressure simultaneously, and therefore would not affect the phenomenon we describe.

In the model, we assume that ATP diffuses freely; however, there is evidence that this assumption may not be appropriate. ${ }^{65,66}$ A more accurate scenario may be that the cells' energy demands are orchestrated by the creatine-kinase phosphotransfer system. ${ }^{67,68}$ In the myofibrils, ATP hydrolysis produces energy (for AAT) and ADP. To make more ATP, the ADP is rephosphorylated by creatine-phosphate (CP), which diffuses to the myofibrils from the mitochondria. This scenario suggests that AAT relies on CP diffusion, rather than ATP diffusion; however, the PNIFF would also affect the diffusion of CP in a similar way to ATP, and would therefore lead to a depletion of 
$\mathrm{CP}$ in the same region as we suggested for ATP (as their diffusion coefficients are similar). Therefore, the PNIFF mechanism would still lead to an energy deficiency that would interrupt AAT.

To determine whether our proposed mechanism of axonal cell death occurs in glaucoma, empiric experimental testing should be performed; however, such testing is very challenging because of the difficulties involved in measuring pressure gradients within tissues. Incompressible tissues like the optic nerve are especially difficult to study in this way because small leaks can have a large effect on the measured pressures. To date, the best attempt at measuring pressure within the optic nerve has been the work of Morgan et al. ${ }^{69}$; however, they were unable to completely eliminate the problems associated with blockage of the fine hollow needle to which the pressure transducer was attached or the problems arising from leakages induced by the insertion of the needle. Despite these difficulties, they were able to demonstrate three important features of a dog model of glaucoma. They showed that: (1) there is a pressure gradient centered on the lamina cribrosa (with the pressure reducing as they moved from the optic nerve head toward the synaptic terminal), (2) the pressure in the optic nerve sheath is the same as the CSF pressure, and (3) the pressure gradient is determined by both the CSF and intraocular pressures.

One interesting feature of the work of Morgan et al. ${ }^{69}$ is that they passed the needle obliquely through the optic nerve. Our model predicts that the pressure in the optic nerve will vary both longitudinally and radially; hence, it would be instructive to repeat these experiments but to alter the course of the needle to allow longitudinal and radial pressure changes to be measured independently. Of even greater interest would be to measure the intraneuronal and interstitial fluid flows; however, such measurements are even more challenging and have not been attempted to date.

In contrast to a glaucomatous raised intraocular pressure, an increase in CSF pressure can also occur clinically, giving rise to a condition called papilledema. Chronic papilledema (in conditions such as benign intracranial hypertension) leads to progressive death of retinal ganglion cells, with cells in the periphery of the optic nerve preferentially affected. Our model can readily be modified to account for the reversed pressure gradient presented in papilledema, and predicts a reversed pressure distribution with a steep increase in pressure at the periphery of the optic nerve rather than the steep decrease predicted in the glaucomatous case. The increasing pressure gradient could also produce a PNIFF and failure of orthograde AAT, suggesting that the PNIFF mechanism may potentially be relevant to papilledema.

This article suggests a potential mechanism to explain why raised intraocular pressure leads to optic neuropathy. It should be noted that the proposed PNIFF theory is compatible with both the mechanical and the vascular theories, and that these mechanisms are not mutually exclusive. The mathematical model presented makes clear predictions which, while experimentally challenging, are testable.

\section{References}

1. Ethier CR, Johnson M, Ruberti J. Ocular biomechanics and biotransport. Annu Rev Biomed Eng. 2004;6(1):249-273.

2. Fechtner RD, Weinreb RN. Mechanisms of optic nerve damage in primary open angle glaucoma. Surv Opbthalmol. 1994;39(1):23-42.

3. Schumer RA, Podos SM. The nerve of glaucoma! Arch. Opbthalmol. 1994;112(1):37-44.

4. Yan DB, Coloma FM, Metheetrairut A, Trope GE, Heathcote JG, Ethier CR. Deformation of the lamina cribrosa by elevated intraocular pressure. Br J Ophthalmol. 1994;78(8):643-648.

5. Burgoyne CF, Downs JC, Bellezza AJ, Suh J-KF, Hart RT. The optic nerve head as a biomechanical structure: a new paradigm for understanding the role of IOP-related stress and strain in the pathophysiology of glaucomatous optic nerve head damage. Prog Retin Eye Res. 2005;24:39-73.

6. Yamamoto T, Kitazawa Y. Vascular pathogenesis of normal-tension glaucoma: a possible pathogenetic factor, other than intraocular pressure, of glaucomatous optic neuropathy. Prog Retin Eye Res. 1998;17(1):127-143.

7. Downs JC, Yang H, Girkin C, et al. Three-dimensional histomorphometry of the normal and early glaucomatous monkey optic nerve head: neural canal and subarachnoid space architecture. Invest Ophthalmol Vis Sci. 2007;48(7):3195-3208.

8. Yang H, Downs JC, Girkin C, et al. 3-D histomorphometry of the normal and early glaucomatous monkey optic nerve head: lamina cribrosa and peripapillary scleral position and thickness. Invest Opbthalmol Vis Sci. 2007;48(10):4597-4607.

9. Yang H, Downs JC, Bellezza A, Thompson H, Burgoyne CF. 3-D histomorphometry of the normal and early glaucomatous monkey optic nerve head: prelaminar neural tissues and cupping. Invest Ophthalmol Vis Sci. 2007;48(11):5068-5084.

10. Sigal IA, Flanagan JG, Terinegg I, Ethier CR. Finite element modeling of optic nerve head biomechanics. Invest Ophthalmol Vis Sci. 2004;45(12):4189-4199.

11. Sigal IA, Flanagan JG, Ethier CR. Factors influencing optic nerve head biomechanics. Invest Opbthalmol Vis Sci. 2005;46(11): $4189-4199$.

12. Dumskyj MJ, Eriksen JE, Doré CJ, Kohner EM. Autoregulation in the human retinal circulation: assessment using isometric exercise, laser Doppler velocimetry, and computer-assisted image analysis. Microvasc Res. 1996;51(3):378-392.

13. Rassam SM, Patel V, Chen HC, Kohner EM. Regional retinal blood flow and vascular autoregulation. Eye. 1996;10(3):331-337.

14. Flammer J, Orgül S. Optic nerve blood-flow abnormalities in glaucoma. Prog Retin Eye Res. 1998;17(2):267-289.

15. Flammer J, Orgül S, Costa VP, et al. The impact of ocular blood flow in glaucoma. Prog Retin Eye Res. 2002;21(4):359-393.

16. Evans DW, Harris A, Garrett M, Chung HS, Kagemann L. Glaucoma patients demonstrate faulty autoregulation of ocular blood flow during posture change. BMJ. 1999;83(7):809-813.

17. Riva CE, Hero M, Titze P, Petrig B. Autoregulation of human optic nerve head blood flow in response to acute changes in ocular perfusion pressure. Graefes Arch Clin Exp. 1997;235(10):618-626.

18. Pillunat LE, Anderson DR, Knighton RW, Joos KM, Feuer WJ. Autoregulation of human optic nerve head circulation in response to increased intraocular pressure. Exp Eye Res. 1997;64:737-744.

19. Weigert G, Findl O, Luksch A, et al. Effects of moderate changes in intraocular pressure on ocular hemodynamics in patients with primary open-angle glaucoma and healthy controls. Opbthalmology. 2005;112(8):1337-1342.

20. Hafez AS, Bizzarro RLG, Lesk MR. Evaluation of optic nerve head and peripapillary retinal blood flow in glaucoma patients, ocular hypertensives, and normal subjects. Am J Opbthalmol. 2003; 136(6):1022-1031

21. Morgan JE. Optic nerve head structure in glaucoma: astrocytes as mediators of axonal damage. Eye. 2000;14:437-444.

22. Hernandez MR, Agapova OA, Yang $P$, et al. Differential gene expression in astrocytes from human normal and glaucomatous optic nerve head analyzed by cDNA microarray. Glia. 2002;38(1):45-64.

23. Lukas TJ, Miao HX, Chen L, et al. Susceptibility to glaucoma: differential comparison of the astrocyte transcriptome from glaucomatous African American and Caucasian American donors. Genome Biol. 2008;9(7):R111.

24. Anderson DR, Hendrickson A. Effect of intraocular pressure on rapid axoplasmic transport in monkey optic nerve. Invest $O p b$ thalmol Vis Sci. 1974;13(10):771-783.

25. Balaratnasingam C, Morgan WH, Bass L, Matich G, Cringle SJ, Yu DY. Axonal transport and cytoskeletal changes in the laminar regions after elevated intraocular pressure. Invest Ophthalmol Vis Sci. 2007;48(8):3632-3644.

26. Minckler DS, Bunt AH, Johanson GW. Orthograde and retrograde axoplasmic transport during acute ocular hypertension in the monkey. Invest Opbthalmol Vis Sci. 1977;16(5):426-441.

27. Martin KRG, Quigley HA, Valenta D, Kielczewski J, Pease ME. Optic nerve dynein motor protein distribution changes with in- 
traocular pressure elevation in a rat model of glaucoma. Exp Eye Res. 2006;83(2):255-262.

28. Quigley HA, Anderson DR. The dynamics and location of axonal transport blockage by acute intraocular pressure elevation in primate optic nerve. Invest Ophthalmol Vis Sci. 1976;15(8):606-616.

29. Levy NS. The effects of elevated intraocular pressure on slow axonal protein flow. Invest Ophthalmol Vis Sci. 1974;13(9):691-640.

30. Vallee RB, Williams JC, Varma D, Barnhart LE. Dynein: An ancient motor protein involved in multiple modes of transport. $J$ Neurobiol. 2004;58(2):189-200.

31. Jonas JB, Muller-Bergh JA, Schlotzer-Schrehardt UM, Naumann GO. Histomorphometry of the human optic nerve. Invest Ophthalmol Vis Sci. 1990;31(4):736-744.

32. Mikelberg FS, Drance SM, Schulzer M, Yidegiligne HM, Weis MM. The normal human optic nerve; axon count and axon diameter distribution. Ophthalmology. 1989;96(9):1325-1328.

33. Keener J, Sneyd J. Mathematical Physiology. New York: Springer; 2004.

34. Quigley HA. Neuronal death in glaucoma. Prog Retin Eye Res. 1999;18(1):39-57.

35. Shou T, Liu J, Wang W, Zhou Y, Zhao K. Differential dendritic shrinkage of and retinal ganglion cells in cats with chronic glaucoma. Invest Ophthalmol Vis Sci. 2003;44(7):3005-3010.

36. Morgan JE. Retinal ganglion cell shrinkage in glaucoma. $J$ Glaucoma. 2002;11(4):365.

37. Haak RA, Kleinhans FW, Ochs S. The viscosity of mammalian nerve axoplasm measured by electron spin resonance. J Physiology. 1976;263(2):115-137.

38. Spyropoulos CS. Water fluxes in a nerve fiber. J Membrane Biol. 1977;32(1):1-18

39. Vargas FF. Filtration coefficient of the axon membrane as measured with hydrostatic and osmotic methods. J Gen Physiol. 1968; 51(1):13-27.

40. Wallin BG. Water permeability in resting and stimulated crayfish nerve. J Gen Physiol. 1969;54(4):462- 478.

41. Rostovtseva TK, Bezrukov SM. ATP transport through a single mitochondrial channel, VDAC, studied by current fluctuation analysis. Biophys J. 1998;74(5):2365-2373.

42. Leal LG. Laminar flow and convective transport processes: scaling principles and asymptotic analysis. Boston, USA: ButterworthHeinemann; 1992.

43. Luby-Phelps K, Mujumdar S, Mujumdar RB, Ernst LA, Galbraith W, Waggoner AS. A novel fluorescence ratiometric method confirms the low solvent viscosity of the cytoplasm. Biophys J. 1993;65(1): 236-242.

44. Fushimi K. Low viscosity in the aqueous domain of cell cytoplasm measured by picosecond polarization microfluorimetry. J Cell Biol. 1991;112(4):719-725.

45. Valberg PA. Cytoplasmic motions, rheology, and structure probed by a novel magnetic particle method. J Cell Biol. 1985;101(1):130-140.

46. Dix J, Verkman A. Mapping of fluorescence anisotropy in living cells by ratio imaging: application to cytoplasmic viscosity. Biophys J. 1990;57(2):231-240.

47. Wandelt B, Cywinski P, Darling GD, Stranix BR. Single cell measurement of micro-viscosity by ratio imaging of fluorescence of styrylpyridinium probe. Biosens Bioelectron. 2005;20(9):1728-1736.

48. Burns VW. Microviscosity and calcium exchange in yeast cells and effects of phenethyl alcohol. Exp Cell Res. 1971;64(1):35-40.

49. Burns VW. Measurement of viscosity in living cells by a fluorescence method. Biochem Biophys Res Commun. 1969;37(6): $1008-1014$.

50. Chevalier-Larsen E, Holzbaur ELF. Axonal transport and neurodegenerative disease. Biochim Biophys Acta. 2006;1762(11-12): $1094-1108$

51. Reid E, Kloos M, Ashley-Koch A, et al. A kinesin heavy chain (KIF5A) mutation in hereditary spastic paraplegia (SPG10). Am J Hum Genet. 2002;71(5):1189-1194.

52. Lo Giudice M, Neri M, Falco M, et al. A missense mutation in the coiled-coil domain of the KIF5A gene and late-onset hereditary spastic paraplegia. Arch Neurol. 2006;63(2):284-287.

53. Morgan JE. Circulation and axonal transport in the optic nerve. Eye. 2004;18:1089-1095.
54. Quigley HA, McKinnon SJ, Zack DJ, et al. Retrograde axonal transport of BDNF in retinal ganglion cells is blocked by acute IOP elevation in rats. Invest Ophthalmol Vis Sci. 2000;41(11):3460-3466.

55. Pease ME, McKinnon SJ, Quigley HA, Kerrigan-Baumrind LA, Zack DJ. Obstructed axonal transport of BDNF and its receptor TrkB in experimental glaucoma. Invest Ophthalmol Vis Sci. 2000;41(3): $764-774$.

56. Votruba M. Molecular genetic basis of primary inherited optic neuropathies. Eye. 2004;18:1126-1132.

57. Duke-Elder S, Wybar K. The anatomy of the visual system. Vol. 2. System of Ophthalmology. St. Louis: CV Mosby; 1961.

58. Sajjadi SA, Harirchian MH, Sheiklibahaei N, et al. The relation between intracranial and intraocular pressures: study of $50 \mathrm{pa}-$ tients. Ann Neurol. 2006;59(5):867-870.

59. Niesel P, Flammer J. Correlations between intraocular pressure, visual field and visual acuity, based on 11 years of observations of treated chronic glaucomas. Int Ophthalmol. 1980;3(1):31-35.

60. Chauhan BC, Drance SM. The relationship between intraocular pressure and visual field progress in glaucoma. Graefes Arch Clin Exp. 1992;230:521-526.

61. Weber J, Kroll W, Krieglstein GK. Intraocular pressure and visual field decay in chronic glaucoma. Ger J Ophthalmol. 1993;2:165-169.

62. Martínez-Belló C, Chauhan BC, Nicolela MT, McCormick TA, LeBlanc RP. Intraocular pressure and progression of glaucomatous visual field loss. Am J Ophthalmol. 2000;129(3):302-308.

63. Berdahl JP, Allingham RR, Johnson DH. Cerebrospinal fluid pressure is decreased in primary open-angle glaucoma. Ophthalmology. 2008;115(5):763-768.

64. Teng C, Gurses-Ozden R, Liebmann JM, Tello C, Ritch R. Effect of a tight necktie on intraocular pressure. BMJ. 2003;87(8):946-948.

65. Selivanov VA, Krause S, Roca J, Cascante M. Modeling of spatial metabolite distributions in the cardiac sarcomere. Biophys J. 2007; 92(10):3492-3500

66. Vendelin M, Eimre M, Seppet E, et al. Intracellular diffusion of adenosine phosphates is locally restricted in cardiac muscle. $\mathrm{Mol}$ Cell Biochem. 2004;256(1):229-241.

67. Suarez RK. Shaken and stirred: muscle structure and metabolism. J Exp Biol. 2003;206(12):2021-2029.

68. Dzeja PP, Terzic A. Phosphotransfer networks and cellular energetics. J Exp Biol. 2003;206(12):2039-2047.

69. Morgan WH, Yu D-Y, Cooper RL, et al. The influence of cerebrospinal fluid pressure on the lamina cribrosa tissue pressure gradient. Invest Ophthalmol Vis Sci. 1995;36(6):1163-1172.

\section{Appendix A}

\section{Derivation of Governing Equation in the Optic Nerve}

We approximate the cross section of the optic nerve by a hexagonal lattice of axons, a small part of which is shown in Figure A1. We use a Cartesian coordinate system $(x, y)$, and let $2 R$ denote the distance between the centers of two adjacent axons. The axons are labeled by the $(x, y)$ coordinate of their central axis, and we assume that the pressure in a given axon is a function of $z$ only; thus $p_{\left(x_{0}, y_{0}\right)}(z)$ denotes the value of $p_{+}$ in the axon centered on $\left(x_{0}, y_{0}\right)$. The flux per unit area across the axons' membranes equals the net conductivity of the two touching membranes, $\kappa_{+} / 2$, multiplied by the pressure jump across the membranes. For example, referring to Figure A1, the flux out of the axon A into axon B per unit axial distance is

$$
F_{A B}=-\frac{\sqrt{3} R \kappa_{+}}{3}\left(p_{\left(x_{0}+\sqrt{3} R, y_{0}+R\right)}-p_{\left(x_{0}, y_{0}\right)}\right) .
$$

Physiologically $R / a=0.0018$, and it is therefore appropriate to assume that $0<R \ll a$. Using a Taylor series to relate the discrete pressure difference to continuous pressure derivatives, we can approximate the expression for the flux (equation 8), by 


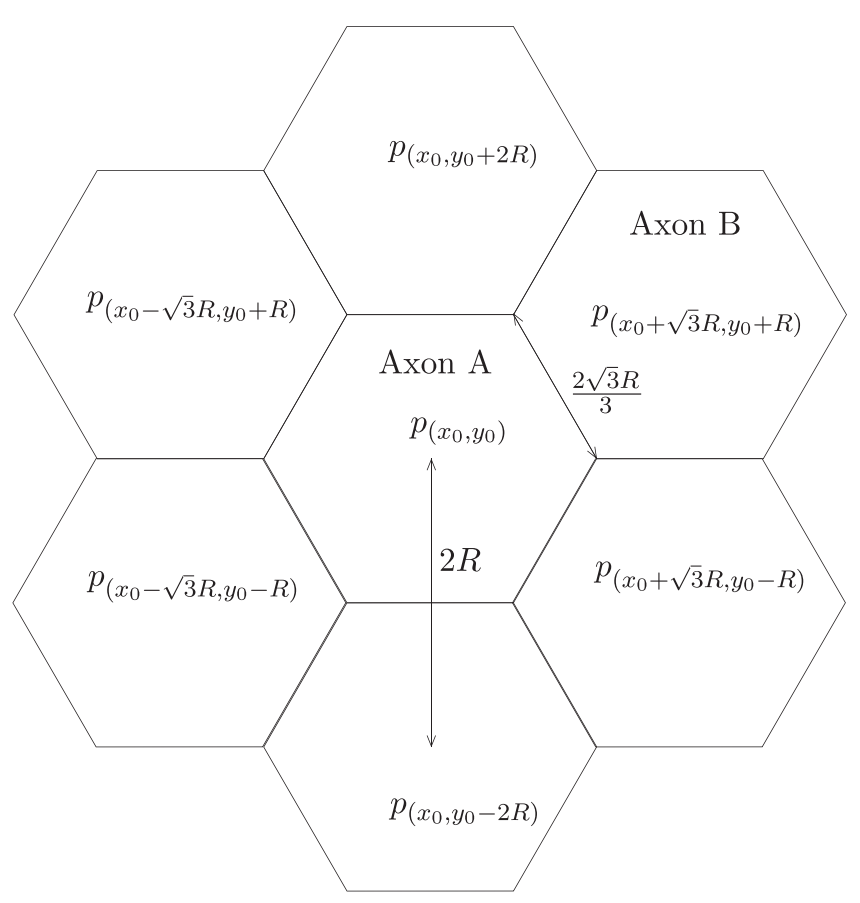

Figure A1. A portion of the optic nerve cross section. The axons are labeled by the $(x, y)$ coordinate of their central axis, and the labels $\mathrm{A}$ and $\mathrm{B}$ are provided to clarify the discussion in Appendix A.

$$
\begin{aligned}
F_{A B} \approx-\frac{\sqrt{3} R \kappa_{+}}{3} & \left(\sqrt{3} R \frac{\partial p_{+}}{\partial x}+R \frac{\partial p_{+}}{\partial y}\right. \\
& \left.+\frac{3 R^{2}}{2} \frac{\partial^{2} p_{+}}{\partial x^{2}}+\sqrt{3} R^{2} \frac{\partial^{2} p_{+}}{\partial x \partial y}+\frac{R^{2}}{2} \frac{\partial^{2} p_{+}}{\partial y^{2}}\right) .
\end{aligned}
$$

Summing the contributions to the flux per unit length in the axial direction through the six walls of axon A (into the surrounding axons) gives an expression for the rate of change of axial flux in a single axon,

$$
\begin{aligned}
& \frac{\partial F}{\partial z}=\frac{\sqrt{3} R \kappa_{+}}{3}\left(p_{\left(x_{0}, y_{0}+2 R\right)}+p_{\left(x_{0}+\sqrt{3} R, y_{0}+R\right)}+p_{\left(x_{0}-\sqrt{3} R, y_{0}+R\right)}\right. \\
& \left.\quad+p_{\left(x_{0}+\sqrt{3} R, y_{0}-R\right)}+p_{\left(x_{0}-\sqrt{3} R, y_{0}-R\right)}+p_{\left(x_{0}, y_{0}-2 R\right)}-6 p_{\left(x_{0}, y_{0}\right)}\right) .
\end{aligned}
$$

As in equation 9, we use a Taylor series to approximate equation 10; this gives

$$
\frac{\partial F}{\partial z} \approx 2 \sqrt{3} R^{3} \kappa_{+}\left(\frac{\partial^{2} p_{+}}{\partial x^{2}}+\frac{\partial^{2} p_{+}}{\partial y^{2}}\right)=\frac{2 \sqrt{3} R^{3} \kappa_{+}}{r} \frac{\partial}{\partial r}\left(r \frac{\partial p_{+}}{\partial r}\right),
$$

where the second equality assumes axisymmetry. As the flow is now in a hexagonal rather than a circular pipe, we rescale the flux in Poiseuille's law (equation 1) by the ratio of the crosssectional areas to give

$$
F(r, z) \approx-\frac{\sqrt{3} R^{4}}{4 \mu} \frac{\partial p_{+}}{\partial z} .
$$

Substituting equation 12 into equation 11 yields equation 4 .

\section{Appendix B}

\section{Solving the Governing Equations}

Nondimensionalization. We introduce dimensionless variables as follows:

$$
p_{ \pm}=p_{\mathrm{c}}+\left(p_{\mathrm{e}}-p_{\mathrm{c}}\right) \hat{p}_{ \pm}, \quad r=a \hat{r}, \quad z=L \hat{z},
$$

and identify three dimensionless parameters

$$
\begin{aligned}
m=\frac{M}{L} \approx 0.10, \quad l_{\mathrm{o}}=\frac{1}{L} \sqrt{\frac{R a^{2}}{8 \mu \kappa_{+}}} & \approx 160, \\
l_{\mathrm{e}} & =\frac{1}{M} \sqrt{\frac{R^{3}}{16 \mu \kappa_{-}}} \approx 0.95,
\end{aligned}
$$

(given to two significant figures), where $m$ denotes the ratio between the length of the axon in the eye and in the brain; $l_{\text {}}$ gives the ratio between a typical axial length scale over which the flux across the axons' membranes influences the intracellular pressure in the optic nerve and the length of the axons in the optic nerve; and finally, $l_{\mathrm{e}}$ denotes the ratio between a typical axial length scale over which the flux across the axons' membranes influences the intracellular pressure in the eye and the length of the axons in the eye. We estimate the dimensionless parameters using the values quoted in Table 1 . As $l_{\mathrm{e}}>m$ and $l_{\mathrm{o}}>1$, we expect the pressure vary slowly along both sections of each individual axon.

We have omitted the circumflexes to simplify the notation. After nondimensionalization, the governing equations 3 and 4 become

$$
\begin{aligned}
& l_{\mathrm{o}}^{2} \frac{\partial p_{+}^{2}}{\partial z^{2}}+\frac{1}{r} \frac{\partial}{\partial r}\left(r \frac{\partial p_{+}}{\partial r}\right)=0 \quad \text { for } \quad z>0 \\
& -m^{2} l_{\mathrm{e}}^{2} \frac{d^{2} p_{-}}{d z^{2}}+p_{-}-1=0 \text { for } z<0
\end{aligned}
$$

and the boundary conditions (equations 5-7), become

$$
\begin{gathered}
\frac{\partial p_{+}}{\partial r}=0 \quad \text { at } \quad r=0, \\
p_{+}=0 \quad \text { at } \quad r=1, \\
\frac{d p_{-}}{d z}=0 \quad \text { at } \quad z=-m, \\
\frac{\partial p_{+}}{\partial z}=0 \quad \text { at } \quad z=1, \\
\left.\frac{p_{-}=p_{+}}{d p_{-}}=\frac{\partial p_{+}}{\partial z}\right\} \quad \text { at } \quad z=0 .
\end{gathered}
$$

The resulting problem (equations 15 and 16) is linear and can be solved by separation of variables.

Solution. Solving equation $15 \mathrm{~b}$ in the eye region subject to the boundary condition in equation $16 \mathrm{c}$, yields

$$
p_{-}(z ; r)=1+A(r)\left(e^{z /\left(l_{e} m\right)}+e^{-(2 m+z) /\left(l_{e} m\right)}\right) \quad \text { for } \quad z<0
$$

where $A(r)$ remains to be determined. With equation 17 , the jump conditions (equation 16e) become

$$
\left.\begin{array}{c}
p_{+}=1+A(r)\left(1+e^{-2 / l_{\mathrm{e}}}\right) \\
\frac{\partial p_{+}}{\partial z}=\frac{A(r)}{l_{\mathrm{e}} m}\left(1-e^{-2 / l_{\mathrm{e}}}\right)
\end{array}\right\} \text { at } z=0,
$$

which we combine to eliminate $A(r)$, giving 


$$
\frac{\partial p_{+}}{\partial z}\left(1+e^{-2 / l_{\mathrm{e}}}\right)=\frac{\left(p_{+}-1\right)}{l_{\mathrm{e}} m}\left(1-e^{-2 / l_{\mathrm{e}}}\right) \text { at } z=0 .
$$

Seeking a solution to equation $15 \mathrm{a}$ for $p_{+}(r, z)$ as a product of a function of $z$ and a function of $r$ leads to the general solution

$$
p_{+}(r, z)=\left(D_{1} e^{\lambda z / l_{0}}+D_{2} e^{-\lambda z / l_{0}}\right)\left[D_{3} J_{0}(\lambda r)+D_{4} Y_{0}(\lambda r)\right]
$$

where $D_{1}, D_{2}, D_{3}$, and $D_{4}$ are constants, and $J_{0}$ and $Y_{0}$ are Bessel functions of the first and second kinds, respectively. Applying equation 16 a gives $D_{4}=0$, equation 16 d gives $D_{2}$ $=D_{1} e^{2 \lambda / l_{0}}$, and equation $16 \mathrm{~b}$ gives $J_{0}(\lambda)=0$. This condition has countably many real roots, and we denote the $j$ th positive real root by $\lambda_{j}$ and let $C_{j}=D_{1} D_{3}$ for the corresponding constants; thus,

$$
p_{+}(r, z)=\sum_{j=1}^{\infty} C_{j}\left(e^{\lambda_{j} z / l_{0}}+e^{\lambda_{j}(2-z) / l_{0}}\right) J_{0}\left(\lambda_{j} r\right)
$$

We obtain the constants, $C_{j}$, by imposing the final boundary condition (equation 19) at $z=0$, which gives

$$
\begin{aligned}
C_{j} & =\left(1-e^{-2 / l_{\mathrm{e}}}\right)\left[\int_{0}^{1} r J_{0}\left(\lambda_{j} r\right) d r\right]\left[\int_{0}^{1} r\left(J_{0}\left(\lambda_{j} r\right)\right)^{2} d r\right]^{-1} \\
& \times\left[\left(1+e^{2 \lambda_{j} / l_{\mathrm{o}}}\right)\left(1-e^{-2 / l_{\mathrm{e}}}\right)-\frac{m l_{\mathrm{e}} \lambda_{j}}{l_{\mathrm{o}}}\left(1+e^{-2 / l_{\mathrm{e}}}\right)\left(1-e^{2 \lambda_{j} / l_{\mathrm{o}}}\right)\right]^{-1} .
\end{aligned}
$$

With equation 18 , the function of integration, $A(r)$, can be calculated via

$$
A(r)=\left[-1+\sum_{j=1}^{\infty} C_{j}\left(1+e^{2 \lambda_{j} / l_{0}}\right) J_{0}\left(\lambda_{j} r\right)\right]\left(1+e^{-2 / \mathrm{l}_{\mathrm{e}}}\right)^{-1}
$$

and hence $p_{-}(z ; r)$ can be determined from equation 17 .

\section{Appendix C}

\section{Modification of the Model to Account for the Finite Thickness of the Lamina Cribrosa}

In the model, we suppose that the lamina cribrosa has negligible thickness; however, in reality its thickness is small but finite. To investigate the effect of a finite-thickness lamina cribrosa, we now consider a variation of the model in which the lamina cribrosa occupies the region between $z=0$ and $z=$ $\delta L$, where $\delta$ is small. Within the lamina cribrosa, we assume that there is no flux through the walls of the axon, which means that in this region $F(r, z)$ is independent of $z$, and hence from equation $1, \partial p / \partial z$ is constant.

After nondimensionalization, the governing equations $15 \mathrm{~b}$ and $16 \mathrm{a}-\mathrm{d}$ are not changed, equation $15 \mathrm{a}$ now applies only in $z>\delta$, and equation $16 \mathrm{e}$ is replaced by the new matching condition

$$
\left.\frac{\partial p_{+}}{\partial z}\right|_{z=\delta}=\left.\frac{d p_{-}}{d z}\right|_{z=0}=\frac{\left.p_{+}\right|_{z=\delta}-\left.p_{-}\right|_{z=0}}{\delta} .
$$

On solving the new system of equations, equation 19 is replaced with

$$
\frac{\partial p_{+}}{\partial z}\left[1+e^{-2 / l_{\mathrm{e}}}+\frac{\delta}{l_{\mathrm{e}} m}\left(1-e^{-2 / l_{\mathrm{e}}}\right)\right]=\frac{p_{+}-1}{l_{\mathrm{e}} m}\left(1-e^{-2 / l_{\mathrm{e}}}\right)
$$

at $z=\delta$. The intracellular pressure in the eye region and optic nerve are still given by equations 17 and 21, respectively, but with coefficients

$$
\begin{aligned}
& C_{j}=\left(1-e^{-2 / l_{\mathrm{e}}}\right)\left[\int_{0}^{1} r J_{0}\left(\lambda_{j} r\right) d r\right]\left[\int_{0}^{1} r J_{0}\left(\lambda_{j} r\right)^{2} d r\right]^{-1}\left[\left(1-e^{-2 / l_{\mathrm{e}}}\right)\right. \\
& \times\left(e^{\lambda_{j} \delta / l_{\mathrm{o}}}+e^{\lambda_{j}(2-\delta) / l_{\mathrm{o}}}\right)-\frac{m l_{\mathrm{e}} \lambda_{j}}{l_{\mathrm{o}}}\left(1+e^{-2 / l_{\mathrm{e}}}\right)\left(e^{\lambda_{j} \delta / l_{\mathrm{o}}}\right. \\
&\left.\left.\quad-e^{\lambda_{j}(2-\delta) / l_{0}}\right)-\frac{\delta \lambda_{j}}{l_{\mathrm{o}}}\left(1-e^{-2 / l_{\mathrm{e}}}\right)\left(e^{\lambda_{j} \delta / l_{\mathrm{o}}}+e^{\lambda_{j}(2-\delta) / l_{\mathrm{o}}}\right)\right]^{-1} \\
& A(r)= {\left[-1+\sum_{j=1}^{\infty} C_{j}\left(e^{\lambda_{j} \delta / l_{\mathrm{o}}}+e^{\lambda_{j}(2-\delta) / l_{\mathrm{o}}}\right) J_{0}\left(\lambda_{j} r\right)\right] } \\
& \times\left[1+e^{-2 / l_{\mathrm{e}}}+\frac{\delta}{l_{\mathrm{e}} m}\left(1-e^{-2 / l_{\mathrm{e}}}\right)\right]^{-1}
\end{aligned}
$$

A lamina cribrosa of thickness $200 \mu \mathrm{m}$ corresponds to $\delta \approx$ 0.002 . Figure $\mathrm{C} 1$ shows that including the finite thickness of the lamina cribrosa has only a small effect on intracellular pressure, and in fact for $\delta<0.01$, the extended model predicts intracellular pressures that are indistinguishable from those given by the original $\delta=0$ model. Therefore, considering a finite-thickness lamina cribrosa will not affect the model's conclusions, that a raised intraocular pressure can create a large enough PNIFF that ATP diffusion could be interrupted.

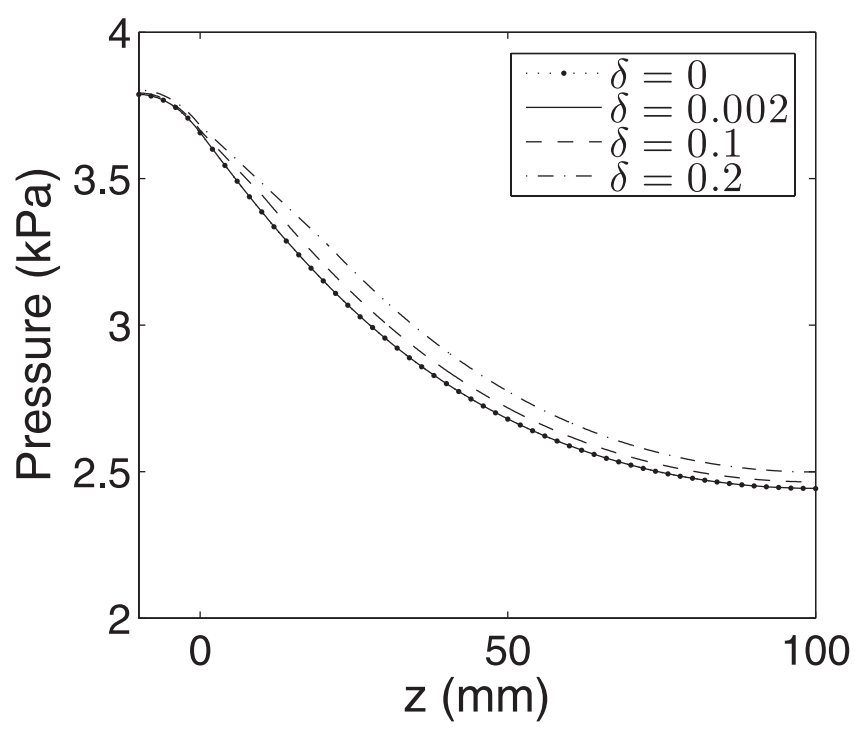

Figure C1. The influence of a finite-thickness lamina cribrosa on the intracellular pressures, $p_{-}(z ; r)$ and $p_{+}(r, z)$. For illustration, the results are shown at a representative radial position close to the edge of the optic nerve, $r=1.595 \mathrm{~mm}$. It is supposed that the lamina cribrosa occupies the region between $z=0$ and $z=\delta L$; pressures are shown for several values of $\delta$. The lines for $\delta=0$ and $\delta=0.002$ are indistinguishable. 